\title{
PERCEPÇÃO, CONHECIMENTO E PRÁTICA DE PEDIATRAS QUANTO AO POSICIONAMENTO DO LACTENTE E O DESENVOLVIMENTO MOTOR
}

\section{PERCEPTION, KNOWLEDGE AND PRACTICE FROM PEDIATRICS ABOUT INFANT PLACEMENT AND MOTOR DEVELOPMENT}

\author{
Priscilla Avelino Ferreira Pinto', Denise Mourão Falci' ${ }^{2}$ Rosane Morais ${ }^{3}$
}

\begin{abstract}
Autora para correspondência: Rosane Morais - rosanesmorais@gmail.com 'Fisioterapeuta. Diamantina, Minas Gerais, Brasil.

${ }^{2}$ Fisioterapeuta. Mestre em Saúde Pública. Belo Horizonte, Minas Gerais, Brasil.

${ }^{3}$ Fisioterapeuta. Doutora em Saúde da Criança e do Adolescente, professora da Universidade Federal dos Vales do Jequitinhonha e Mucuri. Diamantina, Minas Gerais, Brasil.
\end{abstract}

RESUMO I Introdução: O desenvolvimento infantil é uma temática de interesse pediátrico multidisciplinar sendo importante saber qual a percepção de diferentes profissionais envolvidos, considerando que intervenções e orientações por vezes, são necessárias. Objetivo: verificar a percepção, conhecimento e prática de pediatras quanto ao posicionamento do lactente e $\circ$ seu desenvolvimento motor. Método: Estudo qualitativo, do tipo estudo de caso, realizado em uma cidade no interior de Minas Gerais, Brasil. Para a seleção dos participantes da pesquisa utilizou-se a técnica de "amostragem por variedade de tipo", seis pediatras participaram do estudo respondendo uma entrevista individual e semiestruturada, no período de setembro a dezembro de 2014. Os dados foram analisados por "análise de conteúdo" e foram identificadas quatro categorias: orientação quanto ao decúbito do lactente para dormir; percepção do desenvolvimento motor de lactentes após campanha de "supino para dormir"; conhecimento e práticas sobre o desenvolvimento motor dos lactentes; prescrição de prono para o lactente enquanto acordado. Resultados: Alguns pediatras recomendam $\circ$ supino e outros o decúbito lateral para dormir, a última posição principalmente em casos de refluxo gastresofágico. Indicam $\circ$ prono quando acordado principalmente para $\circ$ desenvolvimento do controle cervical e as orientações em relação ao prono foram variadas. Conclusões: Os pediatras entrevistados contraindicam $\circ$ prono, mas principalmente recomendam o decúbito lateral, seguido de supino para dormir. Eles não perceberam, em sua prática clínica, atraso do desenvolvimento motor dos lactentes em decorrência da recomendação de supino para dormir, pelo contrário, consideram as crianças cada vez mais adiantadas em seus marcos do desenvolvimento. $O$ conhecimento e orientações oferecidas aos pais pelos pediatras são fundamentadas na teoria neuromaturacional de desenvolvimento motor.

\begin{abstract}
Introduction: Child development is a topic of multidisciplinary pediatric interest and it is important to know the perception of different professionals, considering interventions and guidelines sometimes necessary for some infants. Objective: To verify the perception, knowledge and practice of pediatricians in the placement of the child and the motor development. Method: Qualitative study of a case study, realized in a city of Minas Gerais, Brasil. For the selection of the participants the technique of "sampling by type variety" was used. Six pediatricians participate in the study, responding to an individual and semi-structured interview, without a period from September to December 2014. The data were analyzed by "content analysis" and four categories were identified: orientation on the supine sleeping infant; perception of the motor development of infants after campaign "sleep in the supine position"; knowledge and practices in the motor development of infants; prone prescription for the child while awake. Results/Conclusion: Some pediatricians recommend the supine and others lateral position for sleeping, the last position especially in cases of gastroesophageal reflux. They do not realize delay in motor development, when recommending supine to sleep, and consider important the positioning of the infant for its development. They indicate the prone when awake primarily for the development of cervical control and guidance to the prone position were varied. The knowledge and guidelines offered to parents by pediatricians are based on the neuromaturational theory of motor development.
\end{abstract}

Keywords: Sudden Infant Death. Child Development. Supine Position. Prone Position.

Palavras-chave: Morte Súbita do Lactente. Desenvolvimento

infantil. Decúbito dorsal. Decúbito ventral 


\section{INTRODUÇÃO}

Devido a um alto número de óbitos de lactentes sem explicação evidente, surgiu a necessidade de se investigar os fatores de risco para a Síndrome da Morte Súbita em Lactente (SMSL), sendo o hábito de dormir em decúbito ventral (prono), um dos fatores mais citados'.

Em 1994, nos Estados Unidos, surgiu a campanha "Back to Sleep", a partir da qual os pediatras passaram a orientar 0 posicionamento dos bebês para dormirem em decúbito dorsal (supino) ${ }^{2}$. No Brasil a primeira campanha mostrando a importância da posição de supino para prevenção da SMSL foi a "Dormir de barriga para cima é mais seguro", lançada em 2009 pela Pastoral da Criança com apoio da Sociedade Brasileira de Pediatria, sendo nacionalmente divulgada na mídia ${ }^{3,4}$.

Entretanto, pesquisas internacionais da área do desenvolvimento infantil alertam para $\circ$ fato de que após as recomendações da posição de supino para dormir, muitos pais não têm deixado seus filhos deitados em prono, nem mesmo durante o dia, favorecendo um atraso no desenvolvimento motor destas crianças $^{5-8}$. Ressalta-se que o desenvolvimento motor nos primeiros anos de vida é importante não apenas para o repertório de movimentação da criança, mas também porque influencia em aquisições nos demais domínios do desenvolvimento infantil, como o cognitivo e a linguagem ${ }^{9,10}$.

Devido a esses estudos, surgiu a campanha internacional "Prone to Play", no ano de 2002, reforçando a orientação de que os lactentes durmam em supino, mas acrescendo a recomendação em colocar os lactentes em prono quando acordados durante $\circ$ dia $^{2,5-6}$. Não foram encontrados registros dessa campanha na literatura brasileira. No entanto, colocar a criança em prono acordado é uma prática pouco frequente de mães brasileiras ${ }^{11}$.

Esta é uma temática de interesse multidisciplinar envolvendo profissionais como terapeutas ocupacionais, fisioterapeutas e enfermeiros, entre outros. No entanto, o médico pediatra é uns dos profissionais da saúde que tem maior contato com - lactente no primeiro ano de vida por meio de consultas de puericultura, as quais têm como foco a prevenção, proteção e promoção da saúde ${ }^{12}$.

Dentro do exposto, é importante conhecer a opinião de pediatras acerca deste tema. Deste modo, o presente estudo teve como objetivo verificar a percepção, conhecimento e prática de pediatras de um município de pequeno porte no interior de Minas Gerais, quanto ao posicionamento do lactente e o seu desenvolvimento motor.

\section{MÉTODO}

Trata-se de um estudo qualitativo, descritivo, do tipo estudo de caso, realizado em um município com cerca de 45.000 habitantes, no interior de Minas Gerais, Brasil. A pesquisa foi aprovada pelo Comitê de Ética em Pesquisa da Universidade Federal dos Vales do Jequitinhonha e Mucuri - UFVJM- (parecer 549.622; CAAE 26515214.1.0000.5108).

Para a seleção dos participantes da pesquisa utilizou-se a técnica de "amostragem por variedade de tipo", desta forma, os critérios de inclusão no estudo foram: ser médico com especialização em pediatria e ter atuação profissional na sede do município pesquisado. Sendo assim, o convite para participar da pesquisa foi feito pessoalmente a todos os nove pediatras do município, os quais se enquadravam nos critérios acima, sendo que seis aceitaram participar do estudo e assinaram o Termo de Consentimento Livre e Esclarecido.

Um roteiro de entrevista estruturada, comum a todos os voluntários, foi aplicado. As perguntas foram: (1) Você considera importante o posicionamento do bebê para o seu desenvolvimento motor? Por quê? (2) Você indica algum posicionamento para os pais de lactentes com o intuito de estimular seu desenvolvimento motor? Qual (is)? (3) Você orienta a posição de prono para os pais como estratégia para o desenvolvimento motor do lactente? (4) A partir de qual idade você considera mais indicada a posição de prono para estimular o desenvolvimento motor da criança? (5) Como você orienta os pais a posicionar seus bebês em prono para estimular o seu 
desenvolvimento motor? (6) Considerando a SMSL, quais têm sido as orientações dadas para os pais de lactentes sobre o posicionamento para dormir? (7) Você acredita que após a Sociedade Americana de Pediatria ter contra indicada a posição de prono para dormir devido a SMSL está havendo atraso no desenvolvimento motor dos lactentes?

As entrevistas foram realizadas no local e horário estabelecido pelos participantes, no período de setembro a dezembro de 2014 . Estas foram sempre realizadas pelo mesmo pesquisador em sala fechada com apenas o entrevistado, para garantir a privacidade do voluntário. As entrevistas foram registradas por meio de um gravador de áudio digital (Sony ICD-BX800). A duração média das entrevistas foi de 5,85 minutos e todas as falas gravadas foram transcritas na íntegra.

As entrevistas foram enviadas para os pediatras por correio eletrônico para que eles pudessem aprovar. Para a garantia do anonimato, os participantes foram identificados como "Pediatra" seguido por um algarismo arábico de um a seis.

Após a transcrição, as entrevistas foram analisadas por meio da técnica de "análise de conteúdo" segundo Bardin (2011)13. A partir dessa técnica foram realizadas leituras flutuantes do conteúdo transcrito e estabelecidas categorias.

\section{RESULTADOS E DISCUSSÃO}

Participaram do estudo seis pediatras, sendo cinco mulheres e um homem com tempo de experiência profissional média de $17,84 \pm 6,3$ anos.

Após a análise dos dados foram identificadas quatro categorias que serão apresentadas e discutidas a seguir: ( 1 ) orientação quanto ao decúbito do lactente para dormir; (2) Percepção do desenvolvimento motor de lactentes após campanha de "supino para dormir"; (3) Conhecimento e práticas sobre o desenvolvimento motor dos lactentes; (4) Prescrição de prono para o lactente enquanto acordado.

\section{Orientação quanto ao decúbito do lactente para dormir.}

Quanto às recomendações do posicionamento para dormir, no presente estudo, o posicionamento em prono foi contraindicado para $\circ$ sono pelos pediatras. Foram encontradas orientações para dormir em supino, mas principalmente o decúbito lateral.

"[...][dormir] sempre de barriguinha para cima. [...]". (Pediatra 6)

“[...] enquanto os pais não estão presentes, decúbito lateral[...]". (Pediatra 2)

"[...] [para dormir] levemente [...] tombadinho, [com] uma colchinha, alguma coisinha [...] de apoio[...]".

(Pediatra 5)

"[...] nunca [dormir] em decúbito ventral, sempre em decúbito dorsal [...] e às vezes levemente inclinado para direita ou para esquerda, mas não totalmente virado[...]". (Pediatra 3)

Estes resultados são semelhantes aos encontrados na literatura brasileira ${ }^{1,14}$. Nunes et al $(2002)^{14}$ verificaram que a posição para dormir mais frequentemente orientada na alta hospitalar em maternidades brasileiras foi o decúbito lateral $(67 \%)$, seguida do supino (15\%). Geib e Nunes (2006) ${ }^{1}$ também observaram, em um estudo populacional sobre hábitos de sono e risco potencial de SMSL, que $\circ$ decúbito lateral foi $\circ$ mais recomentado pelos pediatras $(46,5 \%)$ como posição única para o sono. Uma pequena porcentagem recomendou esta posição alternada com outros decúbitos $(0,7 \% \mathrm{com}$ prono e 0,5\% com supino).

O fato de alguns profissionais da área de saúde e mães de lactentes ainda ficarem receosos em modificar $\circ$ posicionamento de dormir para $\circ$ decúbito dorsal, pode estar relacionado ao medo de que esta posição possa aumentar o risco de complicações, como aspiração de conteúdo gástrico, embora estudos sobre refluxo espontâneo ou induzido sugerem não haver aumento deste risco em decúbito dorsal ${ }^{1,14}$.

Assim, nos primeiros meses de vida do bebê, observou-se que os pediatras entrevistados se preocupavam com o posicionamento no sentido de evitar o refluxo.

"[...] a pediatria preocupa nesse momento mais com o refluxo, o posicionamento por causa das dietas, do que por causa do desenvolvimento motor, o que a gente recomenda muito é a cabeceira um pouco elevada e 
um posicionamento não de lado, ligeiramente tombado para um dos lados [...] Isso logo no recém-nascido [...]"

(Pediatra 5)

Portanto, as respostas dos pediatras do presente estudo corrobora com estudos encontrados na literatura ${ }^{1,14,15}$, ou seja, não indicam o prono para dormir, mas, muitas vezes, indicam o decúbito lateral, embora a posição de supino seja aquela preconizada pela Sociedade de Pediatria para o lactente dormir no intuito de se evitar a $\mathrm{SMSL}^{3,4}$.

\section{Percepção do desenvolvimento motor de lactentes após campanha de "supino para dormir"}

Após a campanha com a recomendação do decúbito dorsal para o sono houve uma redução da prevalência de SMSL nos Estados Unidos, entretanto, surgiu a preocupação de que os pais poderiam estar evitando o prono, mesmo com o bebê acordado, diminuindo as experiências de movimentos nesta posição, em prono e, consequentemente, levando a um possível atraso de marcos motores ${ }^{8-10}$. Não foram encontrados estudos brasileiros sobre esta temática, no entanto, crianças brasileiras apresentam menores escores na progressão em prono no Albert Infant Motor Scale indicando que colocar a criança em prono acordada é uma prática pouco frequente de mães brasileiras ${ }^{11,16}$. Mais estudos são necessários para compreender o motivo desta prática parental pouco frequente.

Os pediatras entrevistados neste estudo relataram não observar, em suas práticas clínicas, atraso do desenvolvimento motor das crianças, pelo contrário, relataram perceber que os bebês estão sendo estimulados cada vez mais cedo e, consequentemente, estão adquirindo mais precocemente os marcos do desenvolvimento neuropsicomotor.

“[...] eu não percebi nada de diferente sobre isso não [...]". (Pediatra 2)

"[...] cada vez mais [os lactentes] estão sendo estimulados, eles estão adquirindo os seus marcos do desenvolvimento neuropsicomotor cada vez mais precoce [...] Os pais cada vez [...] mais ativos, [...] passeiam mais com as crianças, [...] não estão [...] deixando para sair daqui a três meses com o neném [como] antigamente [...] o ritmo moderno dá família [está] cada vez estimulando tanto visual, quanto motor [...]". (Pediatra 3)
Embora ainda seja um tema controverso ${ }^{17}$, os resultados da maioria dos estudos que avaliaram - efeito do sono e/ou posicionamento acordado na idade de aquisição do desenvolvimento motor sugerem que pode haver algum atraso $5-6,8,18-19$.

Kuo et al. $(2008)^{6}$ verificaram que os bebês que ficavam em prono alcançaram mais cedo o marco "arrastar" quando comparados às crianças que não ficavam nessa posição, sugerindo que o tempo em prono pode influenciar as idades de aquisição de marcos motores específicos desta posição. Segundo Majnemer (2007) ${ }^{19}$ os bebês que dormem na posição de decúbito dorsal são susceptíveis a defasagens na aquisição de marcos iniciais, como extensão cervical, rolar e sentar.

Alguns pediatras entrevistados concordam que manter o bebê apenas em supino pode interferir no desenvolvimento motor e a não observância do atraso do desenvolvimento, na prática clínica, foi atribuída às orientações dadas por eles aos pais sobre posicionar do bebê em prono durante o dia.

"[...] não observo isso na minha prática diária porque, por já estar preocupada com isso eu sempre oriento a posição de decúbito ventral como estimulação ao longo do dia, mas se isso não for orientado provavelmente vai

culminar com um atraso, porque [...] os pais por terem medo vão interpretar que a posição é proibida em todos os momentos, o que não é verdade [...]" (Pediatra 6) "[...] acho que, se os pais [...] dão atenção, e pelo menos algumas vezes no dia colocam de bruços não vai haver esse atraso. Por outro lado, se a criança é largada de bruços [...] o dia inteiro [...] esse desenvolvimento também não vai acontecer, porque o estímulo, a atenção, fazem diferença, né [...]" (Pediatra 4)

Alguns pediatras ressaltam que $\circ$ prono deve estar associado à estimulação do bebê para que seja de fato efetiva nesse propósito. De fato, estudos têm demonstrado a importância desta interação da criança com o ambiente no desenvolvimento motor $^{20-23}$. Lobo e Galloway $(2012)^{20}$ encontraram que os bebês de mães que receberam instruções para interagir com seus bebês a partir de dois meses, inclusive em prono, por 15 minutos diários apresentaram habilidades motoras avançadas ao longo do primeiro ano de vida em comparação ao grupo controle.

Portanto, embora alguns estudos internacionais 
tenham encontrado associação entre pouco tempo na posição de prono e atraso no desenvolvimento motor $^{5-8}$, os pediatras do presente estudos afirmam não encontrar esta associação em suas práticas clínicas. São necessários estudos brasileiros para maior compreensão desta temática dentro dos hábitos e práticas parentais. $O$ presente estudo visa fomentar esta discussão.

\section{Conhecimento e práticas sobre o desenvolvimento motor dos lactentes}

Segundo Blank (2003) ${ }^{23}$ os pediatras têm destinado pouco tempo para puericultura e a avaliação mais específica do desenvolvimento neuropsicomotor tem sido realizada apenas para aqueles lactentes de alto risco, como nos casos de prematuridade e asfixia neonatal. Além disto, Gusson e Lopes $(2010)^{24}$ afirmam que o tempo para atendimento de puericultura tem sido reduzido devido à grande demanda destes profissionais. Segundo estes autores, isso se deve ao fato de que a maior parte dos profissionais dependerem dos planos de saúde, tendendo a aumentar o número de atendimento por mês para conseguir um melhor rendimento.

Nunes $(2011)^{25}$ em um estudo qualitativo verificando os conhecimentos e práticas de 26 pediatras sobre $\circ$ desenvolvimento infantil encontrou relatos semelhantes. A autora concluiu que os pediatras dedicavam maior parte da sua consulta na promoção da saúde, em temas biomédicos, como a nutrição e prevenção de doenças. No presente estudo, ao contrário destes estudos os pediatras entrevistados relataram orientar práticas voltadas para o desenvolvimento infantil.

"[...] o estímulo é importante para ele ter maior desenvolvimento motor, [...] é a partir do estímulo que a gente dá, [...] a mudança de posição [...], que ele vai [...] desenvolver esse lado dele motor [...] que é tão natural, mas que precisa de estímulo [...]" (Pediatra 1)

Os pediatras entrevistadosneste estudo consideraram - posicionamento do lactente importante para o seu desenvolvimento motor e aspectos posturais. Acreditam que oportunidades de vivências motoras também favorece 0 desenvolvimento de outros domínios do desenvolvimento, como o cognitivo, estando de acordo com a literatura ${ }^{9-10}$.

"[...] estimulando o motor [...] estimula também o cognitivo. Para fortalecer também [...] a musculatura, [...] para melhorar a sua postura [...] no futuro [...]"

(Pediatra 3)

A respeito das orientações sobre o desenvolvimento motor oferecidas aos pais, observou-se que os pediatras acreditam que para cada fase existem diferentes estímulos a serem oferecidos.

"[...] para cada faixa etária vai ser orientado um posicionamento diferente[...] no primeiro, segundo mês de vida colocar de barriguinha para baixo, para que ele possa estar estimulando o desenvolvimento cervical, em outros momentos de barriga para cima, para que ele possa ter movimentação de membros superiores [...], cada orientação está relacionada a aquisição de um dos marcos do desenvolvimento, e isso vai ao longo do primeiro ano de vida dele [...]" (Pediatra 6)

"[...] ajudar, ensinar a estimulação para ensinar a virar a partir de quatro meses, [...] começar a colocar sentadinho na época de cinco/seis meses. [...] Estimular deixar no chão, deixar andar sozinho segurando nas coisas, no berço, no chiqueirinho, as vezes para ir segurando e subindo sozinho [...]" (Pediatra 2)

Segunda Karasik et al $(2015)^{21}$ o desenvolvimento motor é vítima da suposição da universalidade, ou seja, espera-se que as crianças naturalmente apresentem habilidades motoras em sequência esperada ao longo dos meses. Estes são pressupostos da teoria neuromaturacional, elaborada nas décadas de 1930 e 1940, mas ainda muito difundida no Brasil. Abordagens contemporâneas levam em consideração a oportunidade e tempo gasto de interação espontânea da criança com os componentes existente no ambiente ${ }^{21}$ para 0 surgimento das habilidades motoras. Depende, portanto, da oportunidade de exploração motora oferecida e que, por sua vez, varia conforme crenças e práticas parentais, diversificadas culturalmente ${ }^{22}$.

Embora a literatura indique que pediatras dediquem maior parte da sua consulta em temas biomédicos ${ }^{23,25}$, os pediatras no presente estudo afirmam estar atentos e orientar os pais dos lactentes sobre aspectos do desenvolvimento infantil. Os pressupostos teóricos que embasam estas orientações estão fundamentados na teoria neuromaturacional, embora abordagens ecológicas ${ }^{21}$ têm sido bem difundidas na atualidade. 


\section{Prescrição de prono para o lactente enquanto acordado}

Segundo os pediatras entrevistados a posição de prono é orientada apenas quando o bebê está acordado e sob vigilância dos pais, sendo que o posicionamento em prono como um fator de risco para a morte súbita é uma preocupação presente. Por outro lado, há consciência de que se a criança não for colocada em prono provavelmente ela vai ter um atraso no desenvolvimento.

“[...] quando o neném está sendo vigiado pelos pais, neste momento a gente estimula deixar [na posição de prono][...]" (Pediatra 5)

"[...] Se [a criança] não for colocada nessa posição provavelmente ela vai ter um atraso no desenvolvimento [...]" (Pediatra 6)

As orientações específicas para se colocar o lactente em prono são dadas com o intuito de estimular o controle de cabeça, o movimento dos membros superiores, e para que o mesmo perceba o ambiente por outro ângulo.

"[...] [alguém] estando junto, deixá-lo de bruços para ele ir aprendendo a levantar a cabecinha, para ele ver o mundo de outra forma, [...] depois levantar os bracinhos [...]" (Pediatra 4)

A posição de prono de fato indicada para o desenvolvimento do controle cervical ${ }^{19}$. Salls et al $(2002)^{5}$ encontraram que dentre os lactentes com dois meses de idade que dormiam em prono, $75 \%$ realizou extensão cervical a $45^{\circ}$ e $50 \%$ realizou extensão cervical a $90^{\circ}$. Por outro lado, bebês que não dormiam em prono, mas passavam 15 minutos ou menos em prono quando acordados, $44 \%$ realizou extensão cervical a 45 e $13 \%$ a $90^{\circ}$. Entretanto os autores destacaram que os lactentes que permaneciam mais de 15 minutos por dia tiveram um resultado semelhante com as crianças que dormiam em prono.

No entanto, é importante ressaltar que esta posição favorece $\circ$ alcance de outros marcos do desenvolvimento motor. Estudos demonstram que uma maior exposição a posição prona está associada a capacidade de rolar, arrastar, engatinhar e sentar ${ }^{6,8}$.

Quanto à frequência em colocar o lactente em prono, houve quem relatasse orientar aproximadamente quatro vezes ao dia, utilizando as reações do lactente como parâmetro (por exemplo, choro ou desconforto). Outros relataram não estipular frequência, orientando no momento em que os pais podem dar atenção de qualidade para a criança, e várias vezes ao longo do dia, só evitando os horários durante o sono e logo depois das dietas.

\section{"[...] Frequência assim eu deixo a vontade [...]"}

(Pediatra 2)

"[...] oriento para eles a deixa-los mais ou menos umas quatro vezes ao dia, o máximo que o neném gostar de ficar [...]" (Pediatra 3)

"[...] não adianta nada falar, você tem que fazer isso tantas vezes por dia se não tem ninguém realmente atento aquilo. [...] 0 número de vezes dependia muito, eu trabalhava em unidade básica de saúde, então, eram mães que mal podiam ficar com os filhos, trabalhavam fora, deixavam na creche, [...]. Então

dependia do que elas podiam fazer. Eu sempre valorizava mais isso de qualidade [...]"(Pediatra 4).

De acordo com as teorias contemporâneas de desenvolvimento motor para aquisição de uma determinada habilidade é necessário oportunidade e tempo para a criança adquirir experiência em uma determinada posição ${ }^{20,21}$. Dias de experiência prediz a habilidade da criança de se controlar e fazer decisões adaptativas para ação nas diferentes posições ${ }^{21}$. Desta forma, o importante é que a criança tenha a oportunidade de vivenciar a posição de prono rotineiramente e de forma interativa com o ambiente ${ }^{20}$.

Quando perguntados sobre a partir de qual idade esse posicionamento é indicado, as respostas variaram desde recém-nascido aos seis meses de idade.

"[...] a partir do segundo para o terceiro mês, quando ele [o lactente] começa a ter mais controle cervical, [...] aí você diminui muito esse risco [de morte súbita] [...], sob vigia desde o primeiro momento, a gente pede para que tenha o estímulo [...]" (Pediatra 5) "[...] geralmente no primeiro mês de vida já oriento os pais, [...] não tem essa idade fixa não [...]" (Pediatra 3) "[...] a partir de quatro meses [...]" (Pediatra 2)

"[...] antes [dos 4 meses] tem o problema do refluxo. [...] mais a partir do quinto, sexto mês [...]" (Pediatra 1)

Desta forma, foi possível perceber uma variação 
sobre a idade de iniciar a orientação aos pais acordado. Ressalta-se, no entanto, que a maioria dos estudos que indicam o benefício da posição de prono no desenvolvimento motor iniciam com bebês ainda nos primeiros meses de vida ${ }^{5,6,8,18-20}$.

Assim, o presente estudo apresenta como contribuição fomentar discussão de uma temática ainda pouco explorada na literatura brasileira. O pediatra, por ser um dos profissionais de saúde que apresenta maior contato com bebês no primeiro ano de vida, pode contribuir para que o desenvolvimento motor seja potencializado ao fornecer orientações aos pais durante as consultas médicas sobre o posicionamento da criança.

Admite-se como limitação do estudo a pequena média de tempo das entrevistas, reflexo da dificuldade dos profissionais em disponibilizar tempo para as entrevistas, muitas delas realizadas nos intervalos de descanso entre consultas ou plantões médicos. sobre o decúbito ventral quando o lactente está

\section{AGRADECIMENTOS}

Agradecemos aos pediatras que gentilmente disponibilizaram tempo, conhecimento e percepções acerca do desenvolvimento motor.

\section{CONTRIBUIÇÕES DOS AUTORES}

Pinto PAF foi responsável pela coleta/análise de dados e elaboração do manuscrito; Morais RLS foi responsável pela orientação do projeto, elaboração e revisão do manuscrito; Falci DM foi responsável pela análise dos dados e orientação da metodologia qualitativa empregada no trabalho.

\section{CONFLITOS DE INTERESSES}

Nenhum conflito financeiro, legal ou político envolvendo terceiros (governo, empresas e fundações privadas, etc.) foi declarado para nenhum aspecto do trabalho submetido (incluindo mas não limitandose a subvenções e financiamentos, conselho consultivo, desenho de estudo, preparação de manuscrito, análise estatística, etc).

\section{REFERÊNCIAS}

\section{CONCLUSÃO}

O desenvolvimento infantil é uma temática de interesse pediátrico multidisciplinar e, no primeiro ano de vida o desenvolvimento motor é importante não apenas para o repertório de movimentação da criança, mas também influencia em aquisições nos demais domínios do desenvolvimento infantil.

Os pediatras entrevistados contraindicam o prono, mas principalmente recomendam $\circ$ decúbito lateral, seguido de supino para dormir. Eles não perceberam, em sua prática clínica, atraso do desenvolvimento motor dos lactentes em decorrência da recomendação de supino para dormir, pelo contrário, consideram as crianças cada vez mais adiantadas em seus marcos do desenvolvimento.

Todos consideraram importante o posicionamento do lactente e indicam o prono, porém sob supervisão dos cuidadores.
1. Geib LTC, Nunes ML. Hábitos de sono relacionados à síndrome da morte súbita do lactente: estudo populacional. Cad Saúde Pública. 2006;22(2):415-423. doi: 10.1590/ $\underline{\mathrm{S} 0102-311 \times 2006000200019}$

2. American Academy of Pediatrics. The Changing Concept of Sudden Infant Death Syndrome: Diagnostic coding shifts, controversies regarding the sleeping environment, and new variables to consider in reducing risk. Pediatrics. 2005; $116(5): 1245-55$.

3. Sociedade Brasileira de Pediatria. Bebês devem dormir de barriga para cima [Internet]. 2009. [acesso em 2015 may 26]. Disponível em: http://www.sbp.com.br/arquivo/bebesdevem-dormir-de-barriga-para-cima/

4. Pastoral da Criança. Dormir de barriga pra cima é mais seguro [Internet]. Clóvis Boufleur; 2013. [acesso em 2015 may 26]. Disponível em: http://www.pastoraldacrianca.org. $\mathrm{br} / \mathrm{pt} /$ noticias 2/2251-dormir-de-barriga-para-cima-e-maisseguro

5. Salls JS, Silverman LN, Gatty CM. Brief Report - The Relationship of Infant Sleep and Play Positioning to Motor Milestone Achievement. Am J Occup Ther. 2002;56(5):577580.

6. Kuo YL, Liao HF, Chen PC, Hsieh WS, Hwang AW. The Influence of Wakeful Prone Positioning on Motor Development 
During the Early Life. J Dev Behav Pediatr. 2008;29(5):367376. doi: 10.1097/DBP.0b013e3181856d54

7. Bergman $A B$. Next steps in the study of sudden infant death syndrome. J Pediatr (RioJ). $2011 ; 87(2): 87-88$. doi: 10.2223 /JPED.2076

8. Dudek-Shuriber L, Zelazny S. The Effects of Prone Positioning on the Quality and Acquisition of Developmental Milestones in Four-Month-Old Infants. Pediatr Phys Ther. 2007:48-55. doi: 10.1097/01.pep.0000234963.72945.b1

9. Serdarevic F, Batenburg-Eddes, Mous SE, White T, Hoffman $A$, Jaddoe VWV et al. Relation of infant motor development white nonverbal intelligence, language, comprehension and neuropsychological functioning in childhood: a populationbased study. Dev Sci. 2015;1-13. doi: 10.1111/desc.12326

10. Stapel JC, Hunnius S, Meyer M, Bekkering H. Motor system contribution to action prediction: Temporal accuracy depends on motor experience. Cognition. 2016;148:71-8.

11. Gontijo APB, Magalhães LC, Guerra MQF. Assessing gross motor development of brazilian infants. Pediatr Phys Ther. 2014;26(1):48-55. doi: 10.1097/PEP.0000000000000014

12. Gauterio DP, Irala DA, Cezar-Vaz MR. Puericultura em Enfermagem: perfil e principais problemas encontrados em crianças menores de um ano. Rev Bras Enferm. 201 2;65(3):508-13. doi: 10.1590/S0034$\underline{71672012000300017}$

13. Bardin L. Análise de conteúdo. 6. ed. São Paulo: Edições 70; 2011 . p. 229.

14. Nunes ML, Martins MP, Nelson EAS, Cowan S, Cafferata ML, Costa JC. Orientações adotadas nas maternidades dos hospitais-escola do Brasil, sobre posição de dormir. Cad. Saúde Pública. 2002;18(3):883-886. doi: 10.1590/S0102$\underline{311 \times 2002000300033}$

15. Norton RC, Penna FJ. Refluxo gastroesofágico. J Pediatr. (Rio J.). 2000;76 (Supl.2): S218-S224.

16. Silva PL, Santos DCC, Gonçalves VMG. Influência de práticas maternas no desenvolvimento motor de lactentes do $6^{\circ}$ ao $12^{\circ}$ meses de vida. Rev Bras de Fisioter. 2006;10(2): 225-231.

17. Darrah J, Bartlett D, Maguire TO, Avison WR, LacazeMasmonteil T. Have infant gross motor abilities changed in 20 years? A re-evaluation of the Alberta Infant Motor Scale normative values. Developmental Medicine \& Child Neurology. 2014;56(9): 877-881. doi: $10.1111 /$ dmcn.12452

18. Pérez-Machadoa JL, Rodríguez-Fuentesb G. Relación entre la postura en prono y la adquisición del sostén cefálico a los 3 meses. An Pediatr (Barc). 2013;79(4):241-247. doi: 10.1016/i.anpedi.2013.01.008
19. Majnemer A. Caregiver practices that influence motor development: what are the next moves? Dev Med Child Neurol. 2007;49:804-804.

20. Lobo MA, Galloway, JC. Enhanced Handling and Positioning in Early Infancy Advances Development Throughout the First Year. Child Dev. 201 2; 83(4):1 290-1302. doi: 10.1111/i.1467-8624.2012.01772.x

21. Karasik LB, Tamis-LeMonda CS, Adolph KE, Bornstein MH. Places and postures: A cross-cultural comparasion of sitting in 5-month-olds. J Cross Cult Psycol. 2015;46(8):1023-1038. doi: $10.1177 / 0022022115593803$

22. Bornstein $M H$. Cultural approaches to parenting. Parent Sci Pract. $2012 ; 12$ (2-3): 212-221.

23. Blank D. A puericultura hoje: um enfoque apoiado em evidências. J Pediatr (Rio J). 2003;79(Supl.1):S13-S22.

24. Gusson ACT, Lopes JC. Pediatria no século 21 : uma especialidade em perigo. Rev Paul Pediatr. 2010;28(1):11520. doi: $10.1590 /$ S0103-05822010000100018

25. Nunes C. Pediatricians' Ideas about Child Development and Education. Psicol Reflex Crit. 2011 ;24(4):765-772. doi: $10.1590 /$ S0102-79722011000400017 